\title{
РАСЧЕТНЫЕ МЕТОДЫ ОЦЕНКИ СТЕПЕНИ ОПАСНОСТИ МНОГОВОЛНОВОГО ЛАЗЕРНОГО ИЗЛУЧЕНИЯ
}

\author{
Б. Н. Рахманов, д. т. н., профессор, Московский государственный технический университет им. Н. Э. Баумана, \\ rbn8@yandex.ru; \\ В.И. Кезик, ФГБУ ГНЦ Федеральный медицинский биофизический центр имени А. И. Бурназяна; \\ В. Т. Кибовский, к. т.н., Центр по оценке соответствия и подтверждению качества оборудования, изделий и тех- \\ нологий АНО "АтомТехноТест"; \\ С. В. Тихомиров, д. т. н., ФГУП Всероссийский научно-исследовательский институт оптико-физических измерений
}

\begin{abstract}
Лазерные изделия, генерирующие лазерное излучение одновременно на нескольких длинах волн, широко используются как в гражданской, так и военной сферах деятельности. Гигиеническое нормирование многоволнового лазерного излучения имеет свою специфику. Формулы для вычисления предельно допустимых уровней излучения, регламентированные в отечественных нормативных документах по лазерной безопасности, недостаточно корректны. В статье приведены доказательства необходимости внесения изменений в действующие документы по лазерной безопасности.
\end{abstract}

Статья поступила в редакцию 11.12.2018 Статья принята к публикации 25.03.2019

$\Pi$ рименение лазерных изделий (ЛИз) открывает новые перспективы в развитии экспериментальных научных методов исследований, в частности, в области экспресс-спектроскопии в медицине и экологии. Широко применяются многоволновые Лиз в театрально-зрелищных мероприятиях, например, при проведении лазерных шоу. В военной области применение многоволновых ЛИз позволяет резко повысить эффективность противодействия высокоточным средствам поражения (самонаводящимся зенитным ракетам и снарядам), имеющим оптические каналы наведения и прицеливания, работающие в нескольких спектральных диапазонах. Гигиеническое нормирование многоволнового лазерного излучения (МВ ЛИ) имеет свою специфику. Регламентированные сегодня в отечественных санитарно-гигиенических нормативных документах (СГ НД) по лазерной безопасности формулы для вычисления предельно допустимых уровней (ПДУ) МВ ли недостаточно корректны. Результаты расчетных оценок степени опасности лазерного излучения (СОЛ) многоволновых Лиз, а также вычислений расстояний до границ лазерно опасных зон (ЛО3) для лазерных пучков таких изделий, проведенных с использованием действующих формул вычисления ПДУ МВ ЛИ, приводят к ошибочным результатам и вводят потребителей в заблужде- ние. Оценки СОЛ и расстояний до границ ЛО3 для различных типов современных многоволновых ЛИЗ общетехнического, гражданского и военного назначения, полученные с использованием корректных формул вычисления ПДУ МВ ЛИ, выведенных в работе [1], доказывают необходимость внесения изменений в действующие СГ НД в области лазерной безопасности в части гигиенического нормирования МВ ЛИ.

1. СОВРЕМЕННЫЕ ЛАЗЕРНЫЕ ИЗДЕЛИЯ, ГЕНЕРИРУЮЩИЕ МНОГОВОЛНОВОЕ ЛАЗЕРНОЕ ИЗЛУЧЕНИЕ, И СХЕМЫ МНОГОВОЛНОВОГО ЛАЗЕРНОГО ВОЗДЕЙСТВИЯ НА ГЛАЗА

Уже более 35 лет в нашей стране лазерное излучение отнесено к вредным и опасным физическим факторам воздействия в соответствии с ГОСТ 12.1.040-83 «Система стандартов безопасности труда. Лазерная безопасность. Общие положения" прежде всего из-за способности лазерного излучения, генерируемого в спектральном диапазоне от 380 до 1400 м, нанести непоправимый ущерб зрительному аппарату человека. Оптические среды глаза в этой области спектра прозрачны для ЛИ, которое достигает сетчатки глаза и фокусируется на ней в пятно с крайне малым диаметром около 10 мкм, в котором наблюдается чрезвычайно высокая плотность энергии (мощ- 
ности) ЛИ, превышающая более чем в $10^{5}$ раз ее значение на роговой оболочке глаза [2, 3].

При оценке степени опасности лазерного излучения мы будем в основном принимать во внимание глаз человека, как орган в наибольшей степени подверженный неблагоприятному действию ЛИ.

В последние годы наблюдается заметное возрастание интереса научных, технических и военных специалистов к применению инновационных лазерных технологий. Создаются новые образцы лазерной техники, расширяются области их применения в научных исследованиях, в промышленности, медицине, в рекламном и шоу-бизнесе.

Отмечается значительный прогресс в использовании лазерной техники в военной области. На вооружение российской армии принимаются современные образцы оборонительного нелетального лазерного оружия для целей противовоздушной и противоракетной обороны $[4,5]$. Так, например, президент РФ в своем выступлении перед Федеральным собранием в марте 2018 года продемонстрировал в числе новых образов вооружения мобильный лазерный комплекс "Пересвет" [6] способный решать задачи ПРО и ПВО от низколетящих целей. Комплекс "Пересвет" уже принят на вооружение [7].

Особый интерес как в гражданской, так и военной сфере деятельности, проявляется к лазерным изделиям, способным генерировать ли одновременно на нескольких длинах волн. Обобщенное понятие "лазерное изделие" включает в себя прежде всего непосредственно сам генератор лазерного излучения - лазер. Практически все существующие газовые лазеры и лазеры на парах металлов являются многоволновыми лазерными изделиями. Области применения таких ЛИз весьма разнообразны: от учебных лазеров, используемых для демонстраций и опытов в учебном процессе, до лазеров, разрабатываемых для получения термоядерной энергии .

Многоволновая генерация Ли возможна и в твердотельных ЛИз (лазерах), в которых рабочим телом являются различные кристаллы: $\mathrm{Nd}$ :YAG $\left(\lambda_{1}=1064 \mathrm{Hm}\right), \mathrm{Nd}$ : YLF $\left(\lambda_{1}=1053 \mathrm{нм}\right)$ и др., а генерируемое ими ЛИ подвергнуто нелинейному оптическому преобразованию с помощью других кристаллов: $\mathrm{KTiOPO}_{4}$ (или сокращенно "КTP»), $\mathrm{LiNbO}_{3}$ и др. Лазеры, в которых конструктивно предусмотрено наличие нелинейных оптических преобразователей, принято называть "лазерами с удвоением частоты".
Твердотельные лазеры с удвоением частоты нашли самое широкое применение благодаря их высокому к.п.д. в сочетании с высокой устойчивостью к климатическим и механическим воздействиям и малым габаритам. Это обусловлено тем, что для оптической накачки стали применяться мощные лазерные полупроводниковые диоды (ЛПД), работающие в ближней иК области спектра $(\lambda=800-900$ нм). Лазер с диодной накачкой (DPSS лазер) по существу состоит из двух лазеров: один на основе ЛПД служит для получения энергии накачки, а второй на основе кристалла, являющегося рабочим телом, - для получения узконаправленного монохроматического лазерного пучка с длиной волны $\lambda_{1}$.

Из большого разнообразия решаемых задач следует, что существует постоянно расширяющийся круг лиц как среди научных, технических и военных специалистов, так и среди гражданского населения, контактирующих с многоволновым лазерным излучением.

Отечественные производители лазерной техники предлагают довольно широкий спектр DPSS импульсных лазеров, генерирующих двухволновое и трехволновое лазерное излучение. На рис. 1 показан трехволновый импульсный лазер TECH-263Basic [8]. Этот лазер генерирует импульсно-модулированное лазерное излучение (ИМ ЛИ) с крайне высокой средней мощностью: $\mathrm{P}_{1} \geq 700 \mathrm{MBT}$ при $\lambda_{1}=1053 \mathrm{Hм}, \mathrm{P}_{2} \geq 350$ мВт при $\lambda_{2}=527$ нм, т. е. эти две гармоники выходного Ли в значительной степени опасны для глаз.

Благодаря таким отличительным чертам, как одновременная генерация импульсов на 1053, 527 и 263 нм одним лучом в одном направ-

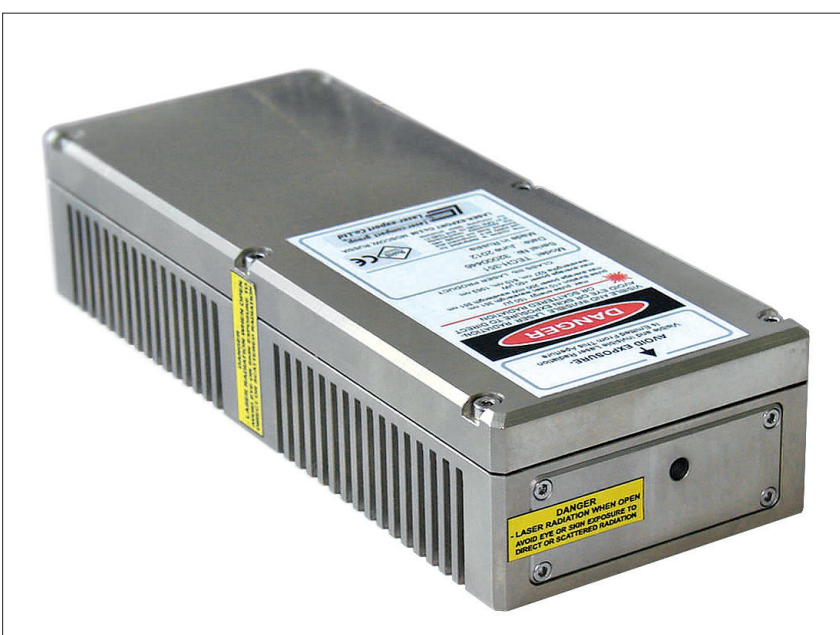

Puc. 1. Многоволновый импульсный пазер TECH-263Basic 


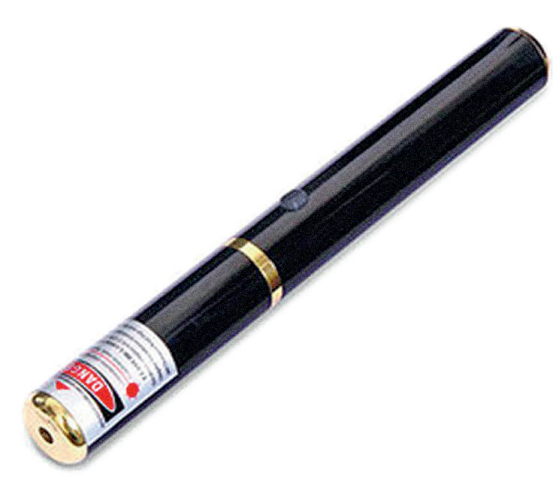

Puc. 2. Лазерная указка "Green laser pointer»

лении и высокой энергии и пиковой мощности в импульсе этот лазер нашел применение в различных видах спектроскопии, лазерной микроскопии, микрообработке материалов, фотоакустике, контрольно-измерительном оборудовании, исследованиях флуоресценции и других научных исследованиях [8].

Именно по схеме DPSS лазера сконструированы современные мощные лазерные целеуказатели, называемые в обиходе лазерными указками (ЛУК), например, распространенная луК марки "Green laser pointer" $(\lambda=532$ нм), показанная на рис. 2 [9].

В реальных лУК на выходе присутствует дополнительно остаточное исходное лазерное ИК-излучение с $\lambda_{1}=1064$ нм. Для повышения безопасности ЛУк в ее конструкции предусмотрен отрезающий ИК-светофильтр, подавляющий побочное ЛИ с $\lambda_{1}=1064$ нм.

Многолетний опыт практического применения ЛУК показал, что нередки случаи повреждения защитных светофильтров (например, разбиваются при падении), при этом основные элементы Лук остаются вполне работоспособными. Неискушенный пользователь видит, что указка продолжает излучать зеленый пучок, и продолжает спокойно использовать ЛУК, не подозревая, что суммарная мощность опасного ли на выходе указки существенно увеличилась. Поэтому луК, работающие как DPSS лазеры с удвоением частоты и имеющие встроенные элементы защиты глаз, мы относим к многоволновым ЛИЗ, представляющим опасность для глаз пользователей и окружающих людей. На рис. 3 показано Лиз военного назначения - переносной лазерный прибор оптико-электронного противодействия ПАПВ [10].
ПАПВ предназначен для обнаружения оптических и оптико-электронных средств (ОЭС), ведущих встречное наблюдение и прицеливание, и постановки им лазерным излучением импульсных световых помех. В режиме постановки помех излучением силового лазера прибор обеспечивает засветку поля зрения (подавление) обнаруженного Оэс с нанесением в отдельных случаях повреждений прицельным сеткам и чувствительным элементам приемных устройств. Согласно тактико-техническим характеристикам ПАПВ, приведенным в [10], следует, что ПАПВ представляет собой по существу трехволновое Лиз.

В последние годы разработаны многоволновые лазерные системы, генерирующие лазерное излучение в дальней ИК-области спектра, предназначенные для противодействия ракетам класса "земля-воздух", угрожающим низколетящим воздушным судам (BC), например, совершающим взлет или посадку. Наибольшую опасность для таких ВС представляют переносные зенитные ракетные комплексы (ПЗРК), например, "Стингер", "Игла". Отмечается, что различными бандформированиями и террористическими организациями в горячих точках широко применяются ПЗРК для поражения летательных аппаратов (ЛА).

Наиболее эффективным средством защиты ВС от зенитных управляемых ракет (ЗУР) с инфракрасными головками самонаведения (ИК ГСН) в настоящее время считаются лазерные системы защиты. "Лазерная система MANTA способна подавлять все типы ИК ГСН ЗУР современных и перспективных ПЗРК" [11]. Основой системы MANTA является автоматическая бортовая лазерная станция постановки помех ALDS (Airborne Laser Damming System), создающая кодированное многоволновое помеховое излучение в широком ИК-диапазоне (рис. 4). При попадании кодированного лазерного излучения на ракету происходит засветка ИК приемника ГСН и в тракте обработки формируется ложный сигнал, приводящий к отклонению рулей ракеты с последующим срывом слежения [11].

Статья [11] была опубликована в 2012 году. В том же году в Интернете были опубликованы материалы, сообщающие об установке лазерных систем защиты, аналогичных системе MANTA, на отечественных спецсамолетах правительственного авиаотряда [12]. В 2016 году появились сообщения об установке лазерных систем защиты от ЗУР на отечественных ВС, эксплуатируемых в зонах ведения боевых действий [13].

Далее рассмотрим ЛИз, специально генерирующие многоволновое ЛИ в видимой области спектра 
19-я Международная

выставка сварочных

материалов, оборудования

weldéx

россварка

и технологий

15-18 октября 2019

Москва, КВЦ «Сокольники»

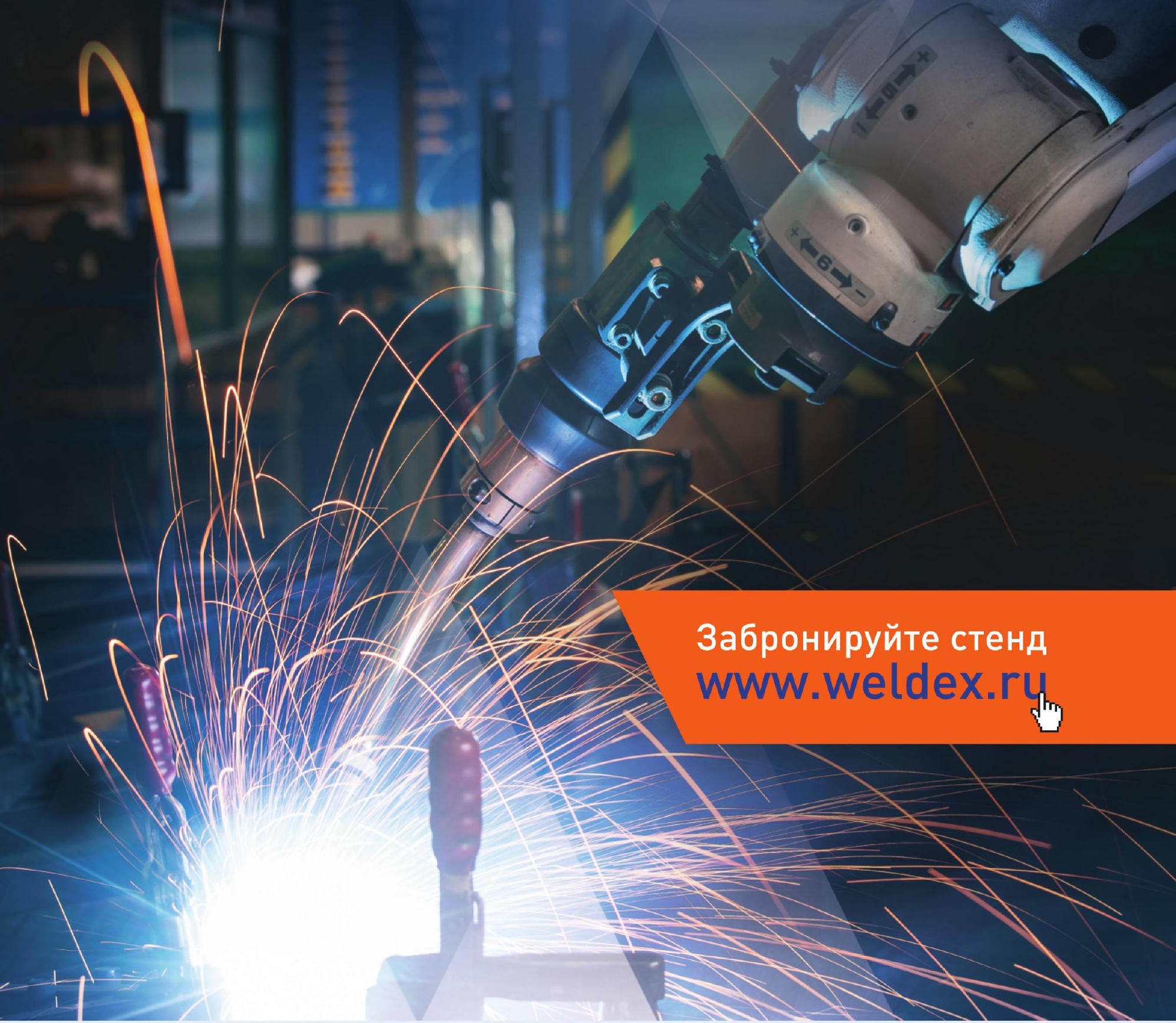




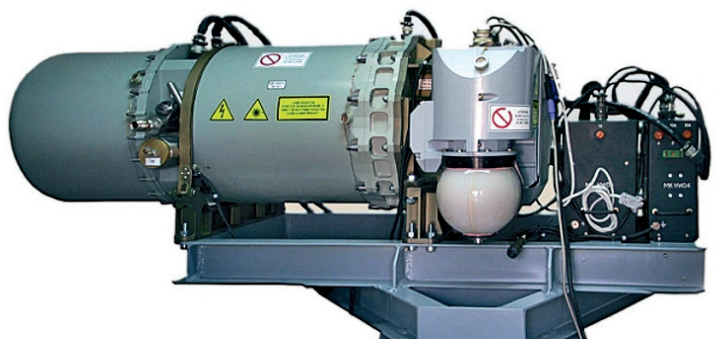

Puc. 4. Автоматическая бортовая пазерная станция постановки помех ALDS

и предназначенные для формирования цветных проекций на поверхностях различных объектов (экранах, стенах зданий и т. п.). Естественно, чем выше мощность ЛИ, генерируемого на различных длинах волн, тем выше качество зрительного восприятия проекций, но и тем выше степень опасности для глаз генерируемых лазерных пучков и даже ли диффузно отраженного от упомянутых поверхностей.

На рис. 5 показан лазерный проектор марки 1500 RGB [14], предназначенный для создания лазерных рекламных проекций и проведения лазерных шоу. Проектор излучает одновременно зеленый, синий и красный лазерные пучки с длинами волн: $\lambda_{1}=520 \mathrm{нм}, \lambda_{2}=450 \mathrm{Hм}, \lambda_{3}=637$ нм. Генерируемые в проекторе лазерные пучки не являются гармониками исходного лазерного ИК-излучения, т.е. не являются пучками, генерируемыми лазером с удвоением частоты. Общая мощность лазерного излучения - 1500 мВт.

Следует предположить, что источниками ЛИ с $\lambda_{1}=520 \mathrm{HM}, \lambda_{2}=450 \mathrm{HM}, \lambda_{3}=637$ нм в рассматриваемом проекторе являются мощные лазерные полупроводниковые диоды, каждый из которых является самостоятельным лиз: ЛИз с излучением на $\lambda_{1}$, ЛИз ${ }_{2}$ с излучением на $\lambda_{2}$ и Лиз ${ }_{3}$ с излучением на $\lambda_{3}$. Лазерный проектор 1500 RGB, как и все другие аналогичные изделия, представляет собой многоволновое лазерное изделие, генерирующее совокупный лазерный пучок, являющийся суперпозицией пучков отдельных ЛИз

Обобщенная схема получения многоволнового лазерного

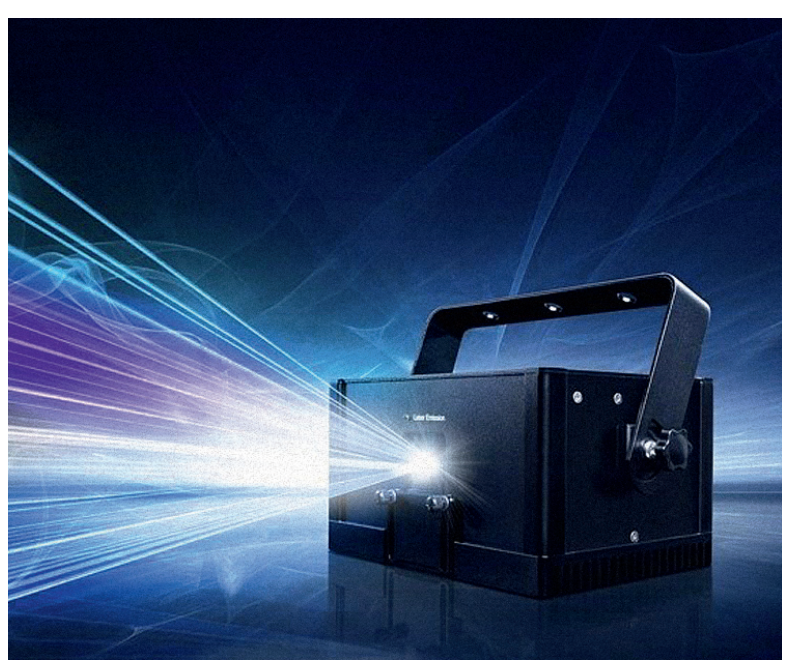

Puc. 5. Лазерный проектор для пазерной рекламы 1500 RGB

пучка, представляющего собой совокупный пучок излучения, генерируемого различными лиз, приведена на рис. 6. Естественно, приведенная на рис. 6 оптическая схема формирования суммарного лазерного пучка является весьма условной.

Заметим, что на схеме (рис. 6) совокупность отдельных ЛИЗ и оптическая система формирования суммарного пучка рассматриваются как совокупное лазерное изделие, обозначаемое как Лиз Совокупное ЛИз $3_{\Sigma}$ может иметь общий защитный кожух с выходным окном для лазерного пучка, как, например, для многоволнового лазерного проектора, но может эксплуатироваться и без кожуха, как, например, открытая многоволновая лазер- 
ная установка для научных исследований. Исходя из изложенного, мы обозначили на рис. 6 защитный кожух пунктирными линиями.

Суммарная мощность выходного лазерного излучения проектора 1500 RGB 1500 мВт является чрезвычайно высокой с позиций обеспечения лазерной безопасности. Лазерное излучение рас-

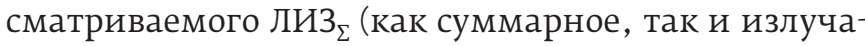
емое на каждой из длин волн) крайне опасно для глаз.

Существуют еще две схемы многоволнового лазерного воздействия на глаз или кожу суммарным излучением отдельных Лиз показано суммарное воздействие ЛИ одновременным действием лазерных пучков, генерируемых группой Лиз, разнесенных в пространстве, а на рис. 7b одновременное облучение зрачка глаза диффузно отраженным лазерным излучением и излучением лазерного пучка.

Возникновение ситуации, показанной на рис. 7а, возможно, например, на строительных площадках, при одновременной работе группы лазерных геодезических приборов (лазерных теодолитов, лазерных нивелиров и т.п.). Рассматриваемая ситуация также возможна при проведении лазерного шоу, в котором используются несколько лазерных проекторов, при проведении военных учений, а также в боевых условиях при использовании противоборствующими сторонами различных лазерных прицелов, дальномеров и систем лазерного противодействия
ОэС. Ситуация одновременного облучения глаза направленным монохроматическим лазерным пучком с длиной волны $\lambda_{1}$ и монохроматическим лазерным излучением с длиной волны $\lambda_{2}$, диффузно отраженным от какой-либо шероховатой поверхности, вполне вероятна при проведении научных исследований на открытых лазерных установках.

\section{2. ОЦЕНКА СТЕПЕНИ ОПАСНОСТИ ИЗЛУЧЕНИЯ МНОГОВОЛНОВЫХ ЛАЗЕРНЫХ ИЗДЕЛИЙ}

Методы расчетной оценки степени опасности лазерного излучения рассмотрены в работе [3]. Методы дозиметрического (инструментального) контроля безопасности ЛИ регламентированы в ГОСТ Р 12.1.031-2010 "Система стандартов безопасности труда. Лазеры. Методы дозиметрического контроля лазерного излучения".

В работе [3] введено понятие "коэффициент степени опасности лазерного излучения (КСОЛ)" коэффициент, показывающий степень превышения уровня энергетической экспозиции (облученности) от лазерного излучения элементарного участка поверхности, на котором расположена заданная точка пространства, над предельно допустимым уровнем (ПДУ) энергетической экспозиции (облученности).

Значения гигиенического норматива лазерного излучения (ПДУ ЛИ) в настоящее время регламентированы на территории РФ в следую-

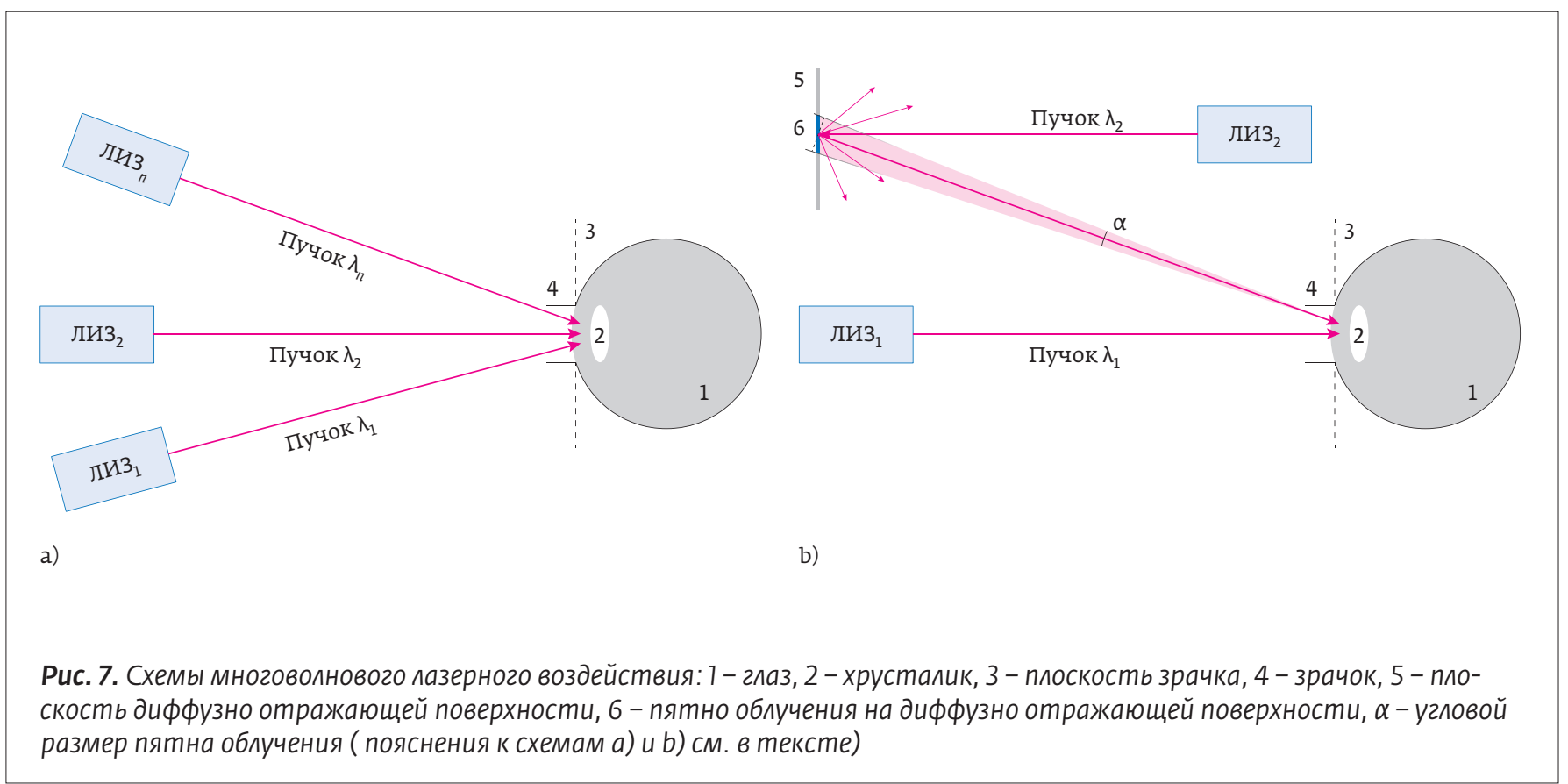

Photonics VOL. 13 № 32019301 
щих санитарно-гигиенических нормативных документах:

- «Санитарные нормы и правила устройства и эксплуатации лазеров" № 5804-91 [15] (далее CH 5804, CH);

- «Санитарно-эпидемиологические правила и нормативы СанПиН 2.2.4.3359-16. Санитарно-эпидемиологические требования к физическим факторам на рабочих местах" (далее СанПиН 2.2.4.3359, СанПиН).

В работе [3] выведена обобщенная формула для вычисления КСОЛ на основе общей схемы взаимодействия оптических полей излучения различных лазерных источников с отдельным участком поверхности тела человека (рис. 8).

Приведем пояснения к схеме (рис. 8). Пусть задана некоторая точка пространства $A$, которую будем называть точкой контроля. При оценке КСОЛ предполагаем, что эта точка находится на поверхности тела (на поверхности кожи или роговой оболочки глаза). Свяжем с т. А некоторый элементарный участок поверхности $d S_{\mathrm{A}}$ и единичный вектор $\bar{n}_{\mathrm{A}}$, направленный по нормали $\mathrm{k} d S_{\mathrm{A}}$.

В общем случае участок поверхности $d S_{\mathrm{A}}$ может облучаться лазерными источниками следующих видов:

- источник лазерного излучения $\mathrm{LS}_{1}$, имеющий меньшие или сопоставимые размеры по сравнению с размерами небольшой области с нормированной площадью $S_{\text {пду }}$, на которой расположен элемент $d S_{\mathrm{A}} ;$ источник $\mathrm{LS}_{1}$ находится на незначительном расстоянии $l_{1}$ от $\mathrm{T}$. А и излучает в небольшом телесном угле; элемент $d S_{\mathrm{A}}$ находится в слабо расходящемся поле излучения (такая модель характерна для случая, когда источником ЛИ является выходное окно лазерного изделия, близко расположенное $\mathrm{K}$ точке $\mathrm{KoH}^{-}$ троля А);

- источник $\mathrm{LS}_{2}$, имеющий примерно одинаковые или бо́льшие размеры по сравнению с размерами области с площадью $S_{\text {Пду }}$; источник $\mathrm{LS}_{2}$ находится на незначительном расстоянии $l_{2}$ от т. А и излучает в сравнительно большом телесном угле; при этом на элемент $d S_{A}$ попадает излучение от всех участков поверхности источника (такая модель характерна для случая, когда источником ЛИ является близко расположенное к т. А сравнительно большое по размерам пятно облучения на диффузно отражающей поверхности);

- источники $\mathrm{LS}_{3}, \mathrm{LS}_{4}$, расстояния $l_{3}, l_{4}$ от которых до т. А значительно превышают размеры источника и площадки $S_{\text {Пду; источники }}$ могут излучать как в узком, так и в широком телесных углах; из-за большой удаленности источников можно считать, что

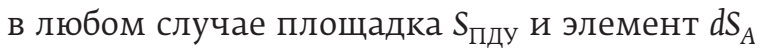
находятся в пучке параллельных лучей (эта модель характерна для источника любого

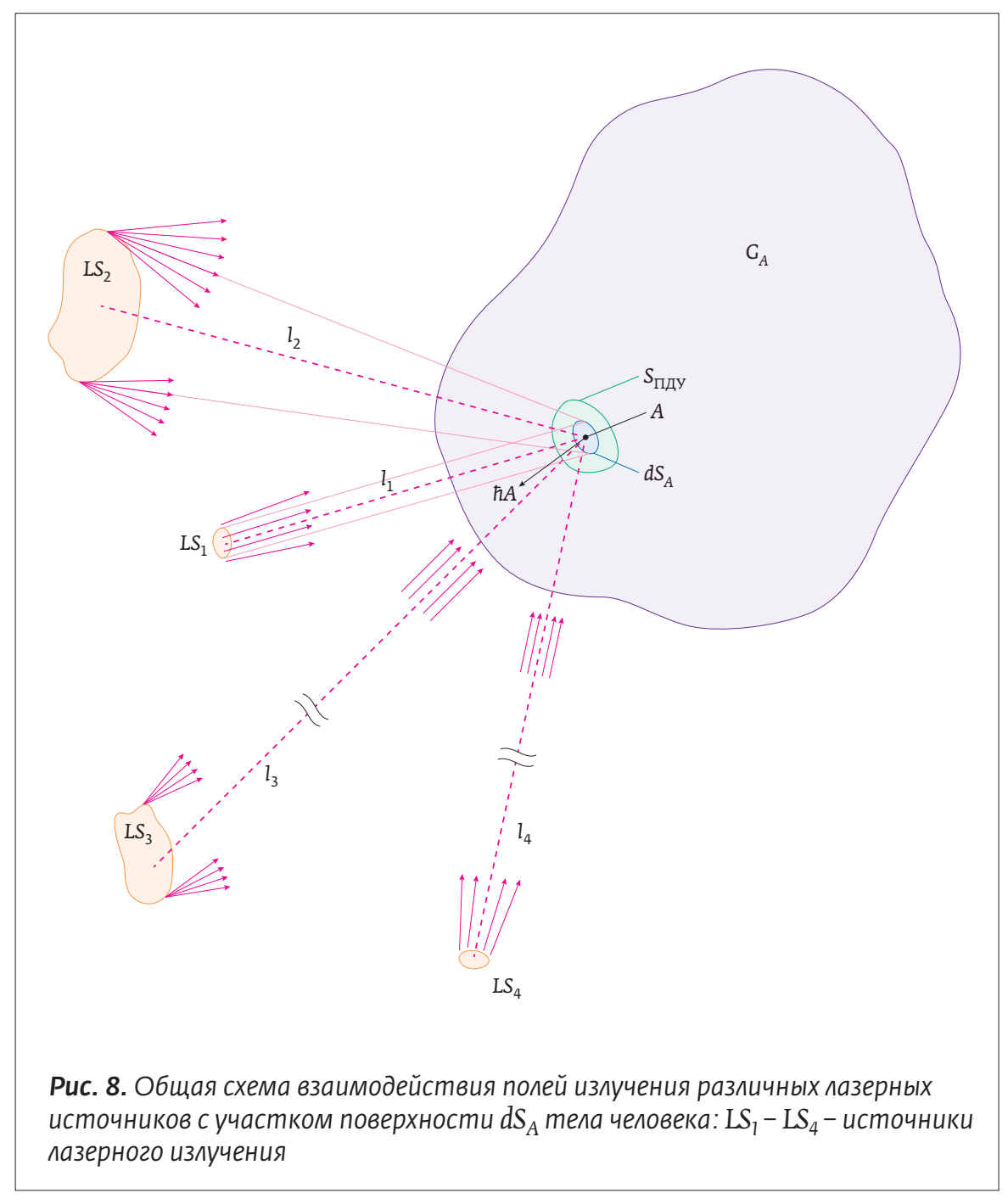


вида, значительно удаленного от т. А, при этом источник ЛИ считается точечным).

Будем считать, что КСОЛ оценивается за некоторое время контроля $\mathrm{T}_{\mathrm{K}}$. Поскольку точка $A$ находится на живом объекте, предполагаем, что в каждый момент времени $t_{\mathrm{K}}$ на интервале $\mathrm{T}_{\mathrm{K}} \mathrm{T}$. $A$ может находиться в различных пространственных положениях, перемещаясь в некоторой области пространства $G_{A}$ из-за естественных движений тела человека (или отдельных частей тела). При этом изменяются взаимные положения направлений лучей, характеризующих распространение излучения источника, и вектора $\bar{n}_{\mathrm{A}}$, а также изменяются расстояния $l_{1}-l_{4}$.

Исходя из общепринятого в технике безопасности принципа учета наихудшего действия воздействующего фактора (принципа учета наибольшего риска), запишем общее выражение для коэффициента степени опасности лазерного излучения $\mathrm{R}_{\text {сол }}$ в следующем виде:

$$
\mathrm{R}_{\text {сол }}=\max _{\mathrm{C}_{a}}\left\{\left[S_{\text {Пду }}^{-1} \iint_{S_{\text {пду }}} H_{A}\left(\mathrm{X}_{A}, \mathrm{Y}_{A}, \mathrm{Z}_{A}, \mathrm{~T}_{\mathrm{K}}\right) d s\right] \mathrm{H}_{\text {ПДУ }}^{-1}\left(t_{\mathrm{B}}, \lambda, \alpha_{L S}\right)\right\},(1)
$$

где $H_{A}\left(X_{A}, Y_{A}, Z_{A}, T_{K}\right)\left(\right.$ Дж• $\left.M^{-2}\right)$ - энергетическая экспозиция элемента $d S_{A}$, окружающего точку контроля A с пространственными координатами $X_{A}, Y_{A}, Z_{A}$, определенная за время контроля $\mathrm{T}_{\mathrm{K}}$ (начало системы координат XYZ, не показанной на рис. 8 будем связывать с цен ${ }^{-}$ тром источника лазерного излучения LS); $\mathrm{H}_{\text {Пду }}\left(t_{\mathrm{B}}, \lambda, \alpha_{\mathrm{LS}}\right)\left(\right.$ Дж· $\left.\mathrm{M}^{-2}\right)$ - ПДУ энергетической экспозиции, зависящий от времени воздействия излучения $t_{\mathrm{B}}(\mathrm{c})$, длины волны излучения $\lambda$ (нм) и углового размера источника излучения $\alpha_{\mathrm{LS}}$ (рад) по отношению к точке контроля;

$S_{\text {пду }}\left(\mathrm{M}^{2}\right)$ - площадь участка поверхности тела человека, значение которой регламентировано в СГ НД, устанавливающих значения ПДу (в соответствии с СН 5804 [15] и СанПиН [17] $S_{\text {Пду }}=0,385 \mathrm{CM}^{2}=3,85 \cdot 10^{-5} \mathrm{M}^{2}$ для роговицы глаза в спектральном диапазоне 380-1400 нм; $S_{\text {пду }}=0,01 \mathrm{~cm}^{2}=10^{-6} \mathrm{M}^{2}$ для кожи и роговицы глаза в спектральных интервалах (СПИ) 180-380 нм и $1400-10^{5}$ нм);

$\max _{\mathrm{GA}_{A}}\{\}$ - обозначает необходимость определения максимального значения параметра, при-

\section{C daeil systems}

\section{Системы активной виброизоляции Настольного типа серия DVIA-T}

- Рабочий диапазон виброизоляции 0.5 - 100 Гц

- Степень виброизоляции > 90\% при 2 Гц

- Время стабилизации менее 0.3 сек

- Грузоподъемность до 150 кг

- Автоматическое самовыравнивание

- Контроль параметров в реальном времени

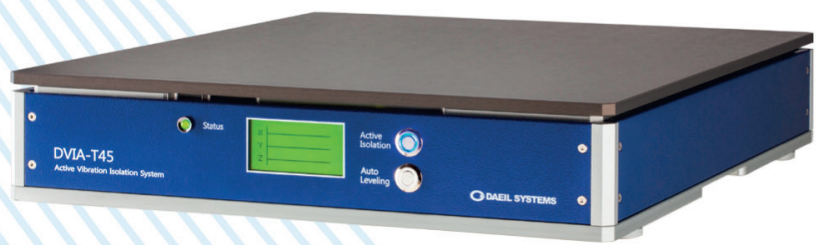

- Атомно-силовая микроскопия

- Сканирующая зондовая микроскопия

- Настольные SEM

(Сканирующие электронные микроскопы)

- Конфокальная микроскопия

- Интерферометрия

- Профилометрия

- Трибология

- Метрология
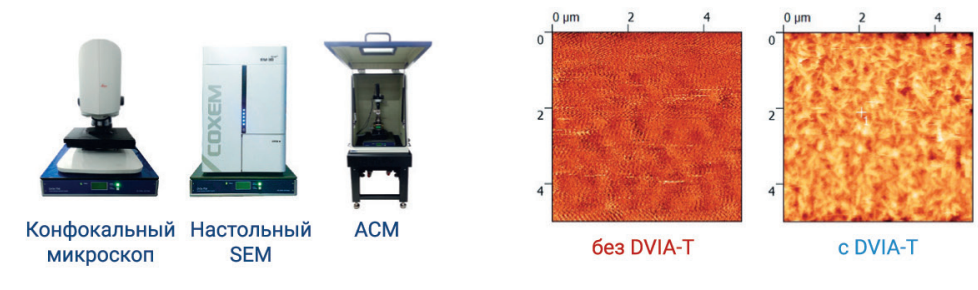

Дистрибьютор в РФ $О 00$ "Промэнерголаб", 105318, Москва, ул. Ткацкая, 1, Тел..+7 (495) 221-12-08, 8 (800) 234-12-08, e-mail: info@czl.ru, www.czl.ru 
веденного в скобках, по всей области $\mathrm{G}_{\mathrm{A}}$ вероятного расположения т. А.

Параметр $\mathrm{H}_{A}\left(\mathrm{X}_{A}, \mathrm{Y}_{A}, \mathrm{Z}_{\mathrm{A}}, \mathrm{T}_{\mathrm{K}}\right)$ представляет собой по сути дозу лазерного излучения, попавшего на элемент $d S_{A}$ за все время контроля $\mathrm{T}_{\mathrm{K}}$. Именно доза падающего излучения наиболее объективно характеризует степень биологического воздействия ЛИ.

В соответствии с СН энергетические параметры ЛИ нормируются для различных временных интервалов в зависимости от режима его генерации. Так, например, для импульсно-модулированного ЛИ (ИМ ЛИ) и для импульсного ЛИ (ИМП ЛИ) с частотой повторения импульсов $\mathrm{F}<0,005$ Гц и $t_{\mathrm{B}}<1$ с в СПИ 380-1400 нм нормируется ПДУ энергии импульса лазерного излучения $W_{\text {ипду }}\left(t_{U}, \lambda, \alpha_{\mathrm{LS}}\right)$ (Дж) длительностью $t_{И}$, прошедшего через круглое отверстие диаметром 7 мм. Это значение преобразуется в ПДУ энергетической экспозиции для импульса излучения $\mathrm{H}_{\text {Ипду }}\left(t_{\mathrm{U}}, \lambda, \alpha_{\mathrm{LS}}\right) \quad$ (Дж· $\mathrm{M}^{-2}$ ) по формуле $H_{\text {Ипду }}\left(t_{U}, \lambda, \alpha_{L S}\right)=W_{\text {ипду }}\left(t_{И}, \lambda, \alpha_{L S}\right) / S_{\text {пду }}$, где $S_{\text {Пду }}=S_{3 \mathrm{P}}=3,85 \cdot 10^{-5} \mathrm{M}^{2}$ - площадь зрачка диаметром 7 мм (средний диаметр зрачка в условиях ночного зрения).

Для непрерывного ЛИ (НЕПР ЛИ) и ИМ ЛИ с частотой повторения импульсов $F \geq 0,005$ Гц и $t_{\mathrm{B}} \geq 1$ с в СПИ 380-1400 нм нормируется ПДУ средней мощности излучения $P_{\text {пду }}\left(t_{\mathrm{B}}, \lambda, \alpha_{\mathrm{LS}}\right)(\mathrm{BT})$, прошедшего через апертуру диаметром 7 мм. Это значение преобразуется в Пду облученности $E_{\text {Пду }}\left(t_{\mathrm{B}}, \lambda, \alpha_{\mathrm{LS}}\right) \quad\left(\mathrm{BT}^{\cdot} \cdot \mathrm{M}^{-2}\right)$ по формуле $E_{\text {Пду }}\left(t_{\mathrm{B}}, \lambda, \alpha_{\mathrm{LS}}\right)=P_{\text {Пду }}\left(t_{\mathrm{B}}, \lambda, \alpha_{\mathrm{LS}}\right) / S_{\text {Пду }}$

Таким образом, для указанных выше случаев нормирования Пду общая формула (1) может использоваться в следующих видах:

$$
\begin{aligned}
& \mathrm{R}_{\text {сол }}=\max _{\mathrm{T}_{\mathrm{K}}}\left\{\max _{\mathrm{C}_{A}}\left\{\begin{array}{c}
{\left[\mathrm{W}_{\text {ИА }}\left(\mathrm{X}_{\mathrm{A}}, \mathrm{Y}_{\mathrm{A}}, \mathrm{Z}_{\mathrm{A}}, \tau_{\mathrm{U}}, t_{\mathrm{K}}\right)\right] \times} \\
\times \mathrm{W}_{\text {ПдУ }}^{-1}\left(\tau_{\mathrm{H}}, \lambda, \alpha_{\mathrm{LS}}\right)
\end{array}\right\}\right\}, \\
& \mathrm{R}_{\text {сол }}=\max _{\mathrm{T}_{\mathrm{K}}}\left\{\max _{\mathrm{C}_{A}}\left\{\left[\begin{array}{c}
\left.S_{\text {Пду }}^{-1} \iint_{S_{\text {Пду }}} H_{\text {ИА }}\left(X_{A}, \mathrm{Y}_{A}, Z_{A}, \tau_{\mathrm{N}}, t_{\mathrm{K}}\right) d s\right] \times \\
\times \mathrm{H}_{\text {Ипду }}^{-1}\left(\tau_{\mathrm{N}}, \lambda, \alpha_{\mathrm{LS}}\right)
\end{array}\right]\right\}\right. \text {, (3) } \\
& \mathrm{R}_{\text {сол }}=\max _{\mathrm{T}_{\mathrm{K}}}\left\{\max _{\mathrm{G}_{A}}\left\{\begin{array}{c}
\left.\left[\mathrm{P}_{A}\left(\mathrm{X}_{\mathrm{A}}, \mathrm{Y}_{\mathrm{A}}, \mathrm{Z}_{\mathrm{A}}, \mathrm{t}_{\mathrm{K}}\right)\right] \times\right] \\
\times \mathrm{P}_{\text {Пду }}^{-1}\left(\tau_{\mathrm{B}}, \lambda, \alpha_{\mathrm{LS}}\right)
\end{array}\right\}\right\},
\end{aligned}
$$

$$
\mathrm{R}_{\text {сол }}=\max _{\mathrm{T}_{\mathrm{K}}}\left\{\max _{\mathrm{C}_{A}}\left\{\left[\begin{array}{c}
\left.S_{\text {Пду }}^{-1} \iint_{S_{\text {пду }}} E_{A}\left(\mathrm{X}_{A}, \mathrm{Y}_{\mathrm{A}}, \mathrm{Z}_{\mathrm{A}}, t_{\mathrm{K}}\right) d s\right] \times \\
\times E_{\text {Пду }}^{-1}\left(\tau_{\mathrm{B}}, \lambda, \alpha_{\mathrm{LS}}\right)
\end{array}\right]\right\}\right. \text {. }
$$

Используя введенное выше понятие "коэффициент степени опасности лазерного излучения", можно проводить специальную оценку условий труда (СОУТ) персонала в условиях воздействия Ли в соответствии с "Методикой проведения специальной оценки условий труда" [17].

Обобщенная схема облучения, приведенная на рис. 8, вполне подходит для решения задачи оценки КСОЛ для многоволнового ЛИ, если предположить, что источники $\mathrm{LS}_{1}-\mathrm{LS}_{4}$ генерируют ЛИ на различных длинах волн $\lambda_{1}-\lambda_{4}$, или в различных СПИ $\Delta \lambda_{1}-\Delta \lambda_{4}$, и воздействуют на элемент $d S_{A}$ одновременно. Схема облучения (рис. 8) охватывает отдельные схемы воздействия, приведенные выше на рис. 1 и 7.

Далее будем использовать более простые формулы для вычисления КСОЛ:

- ДЛя НЕПР ЛИ и ИМ ЛИ с частотой повторения $\mathrm{F} \geq 0,005$ Гц и $t_{\mathrm{B}} \geq 1$ с

$$
\mathrm{R}_{\text {сол }}=\mathrm{P}_{3 \mathrm{P}} / \mathrm{P}_{\text {пду }}=k_{3 \mathrm{P}} \mathrm{P} / \mathrm{P}_{\text {пду }},
$$

где $\mathrm{P}_{3 \mathrm{P}}(\mathrm{BT})=k_{3 \mathrm{P}} \mathrm{P}-$ мощность ЛИ, попадающего в зрачок глаза диаметром $d_{3 \mathrm{P}}=7 \mathrm{Mм}$; $k_{3 Р}$ - коэффициент, показывающий долю мощности Р или энергии $W_{\text {и }}$ лазерного излучения, попадающей в зрачок диаметром $d_{3 \mathrm{P}}=7 \mathrm{Mм}$;

$P_{\text {Пду }}(\mathrm{BT})=P_{\text {пду }}\left(t_{\mathrm{B}}, \lambda, \alpha_{\mathrm{LS}}\right)(\mathrm{BT})$;

- для ИМП ЛИ и ИМ ЛИ с частотой повторения импульсов $\mathrm{F}<0,005$ Гц и $t_{\mathrm{B}}<1$ с

$$
\mathrm{R}_{\text {солш }}=\mathrm{W}_{\text {изг }} / \mathrm{W}_{\text {пду }}=k_{\text {зР }} W_{\text {И }} / \mathrm{W}_{\text {пду }},
$$

где $W_{\text {изр }}\left(\right.$ Дж) $=k_{3 р} W_{\text {и }}$ энергия импульса, попадающего в зрачок диаметром $d_{3 \mathrm{P}}=7 \mathrm{Mм}$; $W_{\text {пду }}\left(\right.$ Дж) $=W_{\text {ипду }}\left(\tau_{\text {И }}, \lambda, \alpha_{\text {LS }}\right)($ Дж).

Для лазерного пучка с равномерным распределением интенсивности в ближней зоне, в которой лазерный пучок целиком проходит через апертуру зрачка, $k_{3 \mathrm{p}}=k_{3 \mathrm{P}}=1$. Для гауссова лазерного пучка $\mathrm{TEM}_{00}$ моды при любом расстоянии Z (м) от плоскости выходного окна ЛИЗ до центра зрачка

$$
k_{3 \mathrm{P}}=k_{3 Р \Gamma}=1-e^{-2 \frac{d_{3 \mathrm{P}}^{2}}{d_{\Pi}^{2}(Z)}},
$$


где $d_{\Pi}(Z)=d_{л и}+10^{3} Z \theta($ мм) - диаметр пучка излучения на расстоянии $Z$ от плоскости выходного окна ЛИЗ;

$d_{л и}($ мм) - диаметр пучка на выходе ЛИЗ, определенный по уровню $1 / \mathrm{e}^{2}$ (коэффициент $10^{3}$ применен для перехода от единиц длины, выраженных в метрах (для расстояния Z) к единицам длины, выраженным в миллиметрах); $\theta$ (рад) - угол расходимости лазерного пучка.

В дальней зоне лазерного пучка, в которой $d_{\Pi} \geq 3,2 d_{3 \mathrm{P}}$,

$$
k_{3 \mathrm{P}}=k_{3 \mathrm{P \Gamma}}=2 d_{3 \mathrm{P}}^{2} d_{\Pi}^{-2}=98 d_{\Pi}^{-2} .
$$

Рассмотрим вопрос определения расстояния до границы лазерно опасной зоны $Z_{\text {лоз для лазер- }}$ ного пучка. В соответствии с СН [16] лазерно опасная зона - часть пространства, в пределах которой уровень лазерного излучения превышает ПДУ. Считаем, что ЛОЗ - это часть пространства, в пределах которой КСОЛ равен или больше единицы. Граница ЛО3 - это поверхность, на которой $\mathrm{R}_{\text {сол }}=1$. Для лазерного пучка лОз ограничена плоскостью сечения, находящейся на расстоянии $Z_{\text {лоз }}$ от выходного окна излучателя. Выведем фор- мулу для вычисления $\mathrm{Z}_{\text {лоз }}$, используя уравнение $\mathrm{R}_{\text {сол }}=k_{\text {зР }}\left(\mathrm{P} / \mathrm{P}_{\text {Пду }}\right)=1$.

Для гауссова лазерного пучка $\mathrm{TEM}_{00}$ моды получаем

$$
\frac{89 \text { P }}{\left(d_{\text {ли }}+10^{3} \theta Z_{\text {лоз }}\right)^{2} \mathrm{P}_{\text {пду }}}=1 .
$$

Решая уравнение (10), получаем

$$
Z_{\text {лоз }}=10^{-3} \theta^{-1}\left(\sqrt{\frac{P}{P_{\text {пду }}}}-d_{\text {ли }}\right) \cdot
$$

Формулы (10), (11) справедливы и для ИМП ли

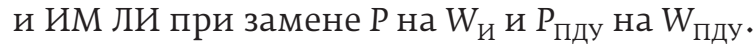

Из формул (6), (7), (11) следует, что для вычис-

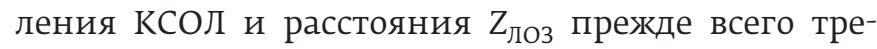

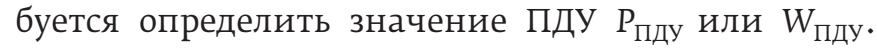
В сравнительно недавно введенных в действие СанПиН 2.2.4.3359 имеется Приложение 8 "правила определения предельно допустимых уровней при одновременном воздействии на глаза и кожу лазерного излучения различных длин волн". При-

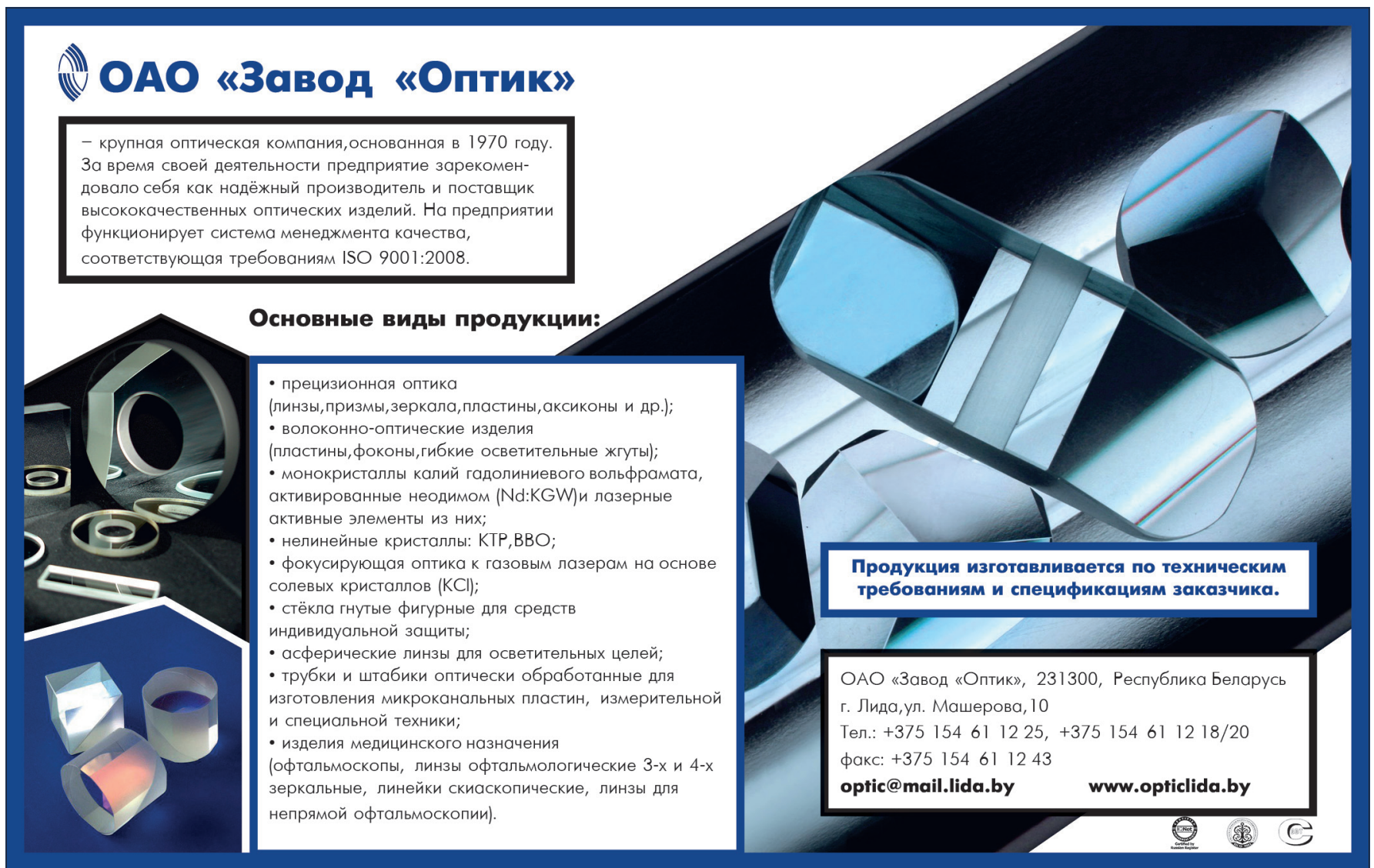


ведем с некоторыми исправлениями извлечения из Приложения 8 [16].

"Степень опасности при одновременном действии излучения различных источников является аддитивной в следующих случаях:

а) воздействие на кожу излучения любых длин волн в диапазоне $180<\lambda \leq 10^{5} \mathrm{Hм}$;

б) воздействие на передние среды глаза излучения в диапазонах длин волн $180<380$ нм и $1400<10^{5} \mathrm{HM}$;

в) воздействие на сетчатку глаза излучения в диапазоне длин волн $380<1400$ нм.

Для каждого из перечисленных трех случаев предельно допустимые уровни устанавливаются независимо.

Предельно допустимая суммарная энергия или мощность излучения от нескольких источников, действие которых является аддитивным, определяется следующими формулами:

$$
\begin{gathered}
W_{\text {пду }}^{\Sigma}=C_{1} W_{\text {Пду }}^{(1)}+\ldots+C_{n} W_{\text {Пду }}^{(n)}=\sum_{i=1}^{n} C_{i} W_{\text {пду }}^{i}, \\
P_{\text {Пду }}^{\Sigma}=C_{1} P_{\text {Пду }}^{(1)}+\ldots+C_{n} P_{\text {пду }}^{(n)}=\sum_{i=1}^{n} C_{i} P_{\text {пду }}^{i}
\end{gathered}
$$

где $n$ - число источников излучения, действие

которых аддитивно;

i - порядковый номер источника;

$W_{\text {пду }}^{\mathrm{i}}, P_{\text {пду }}^{\mathrm{i}},-$ предельно допустимые значения энергии (мощности) лазерного излучения каждого і-го источника;

$W_{\text {пду }}^{\Sigma}, P_{\text {пду }}^{\Sigma}-$ предельно допустимые значения энергии (мощности) суммарного лазерного излучения всех $n$ источников".

Далее в Приложении 8:

" $\mathrm{C}_{\mathrm{i}}$ - относительный энерговклад каждого источника, определяемый как отношение энергии (мощности) источника с порядковым номером i к суммарной энергии (мощности) всех источников, рассчитываемый по формуле:

$$
C_{i}=\frac{W^{i}}{\sum_{i=1}^{n} w^{i}}=\frac{p^{i}}{\sum_{i=1}^{n} p^{i}} .
$$

Оставляя в стороне вопрос о недопустимой небрежности, допущенной при записи условных обозначений в формулах Приложения 8 к СанПиН 2.2.4.3359 [16], зададим более принципиальный вопрос: корректны ли формулы (П8.1), (П8.2) для вычисления значений ПДУ многоволнового лазерного излучения и к чему приводит ошибочность этих формул, доказанная в публикации [1]?

Корректные формулы для вычисления значе-

\begin{tabular}{|c|c|c|c|c|c|c|c|c|c|}
\hline Тип ЛИз & $\lambda_{\mathrm{i}}, \mathrm{HM}^{*}$ & $\begin{array}{c}P_{i}, M B T \\
W_{i}, M Д ж\end{array}$ & $\begin{array}{c}\mathrm{P}^{\Sigma}, \mathrm{MBT} \\
\mathrm{W}^{\Sigma}, \mathrm{MД} \ll\end{array}$ & $C_{i}$ & $\begin{array}{c}P_{\Pi д y,}^{(i)}, \mathrm{MBT} \\
W_{\Pi \text { пду, }}^{(0)} \text { МДж }\end{array}$ & $\begin{array}{c}P_{\Pi д y,}^{\Sigma} \mathrm{MBT}^{*} \\
W_{\Pi д y,}^{\Sigma}, \mathrm{M}^{*}{ }^{*}\end{array}$ & $\begin{array}{c}P_{\Pi д у,}^{\Sigma}, \mathrm{MBT}^{* *} \\
W_{\text {Пду, }}^{\Sigma}, \mathrm{MДж}^{* *}\end{array}$ & $k_{\text {Пду }}$ & $\mathrm{Z}_{\text {ЛОз, }} \mathrm{M}$ \\
\hline $\begin{array}{l}\text { TECH-263 } \\
\text { Basic }\end{array}$ & $\begin{array}{c}527 \\
1053\end{array}$ & $\begin{array}{c}350 \\
700 \mathrm{MBT}\end{array}$ & $1050 \mathrm{MBT}$ & $\begin{array}{l}0,33 \\
0,67\end{array}$ & $\begin{array}{c}0,10 \\
0,35 \mathrm{MBT}\end{array}$ & $0,20 \mathrm{MBT}$ & $0,27 \mathrm{MBT}$ & 1,34 & $\begin{array}{c}236 \\
(203)\end{array}$ \\
\hline $\begin{array}{l}\text { Green } \\
\text { laser } \\
\text { pointer }\end{array}$ & $\begin{array}{c}532 \\
1064\end{array}$ & $\begin{array}{c}100 \\
50 \mathrm{MBT}\end{array}$ & $150 \mathrm{MBT}$ & $\begin{array}{l}0,67 \\
0,33\end{array}$ & $\begin{array}{c}0,10 \\
0,35 \mathrm{MBT}\end{array}$ & $0,13 \mathrm{MBT}$ & $0,18 \mathrm{MBT}$ & 1,38 & $\begin{array}{c}335 \\
(285)\end{array}$ \\
\hline ПАПВ & $\begin{array}{c}532 \\
1064\end{array}$ & $\begin{array}{c}0,7 \\
1,0 \text { мДж }\end{array}$ & 1,7 мДж & $\begin{array}{l}0,41 \\
0,59\end{array}$ & $\begin{array}{c}8 \cdot 10^{-5} \\
10^{-3} \text { мДж }\end{array}$ & $1,8 \cdot 10^{-4}$ мДж & $6,2 \cdot 10^{-4}$ мДж & 3,44 & $\begin{array}{c}317 \\
(171)\end{array}$ \\
\hline ALDS & $\begin{array}{l}2,7-2,9 \\
3,6-4,2\end{array}$ & $\begin{array}{c}1 \cdot 10^{6} \\
1 \cdot 10^{6} \mathrm{MBT}\end{array}$ & $2 \cdot 10^{6} \mathrm{MBT}$ & $\begin{array}{l}0,5 \\
0,5\end{array}$ & $1,5 \cdot 10^{-3} \mathrm{MBT}$ & $1,5 \cdot 10^{-3} \mathrm{MBT}$ & $1,5 \cdot 10^{-3} \mathrm{MBT}$ & 1,0 & $2,3 \cdot 10^{3}$ \\
\hline 1500 RGB & $\begin{array}{l}450 \\
520 \\
637\end{array}$ & $\begin{array}{c}1000 \\
200 \\
300 \mathrm{MBT}\end{array}$ & $1500 \mathrm{MBT}$ & $\begin{array}{l}0,67 \\
0,13 \\
0,20\end{array}$ & $\begin{array}{c}0,10 \\
0,10 \\
0,20 \mathrm{MBT}\end{array}$ & $0,11 \mathrm{MBт}$ & $0,12 \mathrm{MBT}$ & 1,1 & $\begin{array}{l}1155 \\
(1106)\end{array}$ \\
\hline
\end{tabular}
ний ПДУ МВ ЛИ имеют вид [1]:

Результаты вычислений значений Пду и ZлОЗ для многоволновых ЛИз

Аддитивные длины волн (для Лиз марки ALDS значения ПдУ для обоих СПИ одинаковы).

** Значения ПдУ, рассчитанные по действующим формулам (П8.1), (П8.2) из СанПиН 2.2.4.3359.

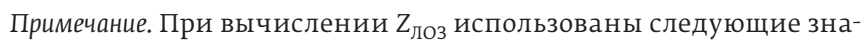
чения параметров $\theta$ и $d_{\text {ли: }}$ :

$3 \cdot 10^{-3}$ рад, 1 мм - для ТЕСН- 263 Ваsic и ПАПВ;

$10^{-3}$ рад, 1 мм - для Green laser pointer и 1500 RGB; $5 \cdot 10^{-3}$ рад, 3 мм - для ALDS. 


$$
P_{\text {ПдУ }}^{\Sigma}=\left[\sum_{i=1}^{n} \frac{c_{i}}{P_{\text {Пमू }}^{(i)}}\right]^{-1}, W_{\text {ПДУ }}^{\Sigma}=\left[\sum_{i=1}^{n} \frac{c_{i}}{W_{\text {ПдУ }}^{(i)}}\right]^{-1} .
$$

Вычислим значения ПдУ МВ ли, генерируемого различными многоволновыми ЛИЗ, рассмотренными в разделе 1. Расчеты проведем по формулам (12) и по формулам (П8.1), (П8.2). Результаты вычислений приведены в таблице.

Там же приведены значения коэффициентов различия $k_{\text {Пду }}=$ ПДУ СанПин $/$ ПДУ кор между ПДУ по СанПиН и корректными значениями ПДУ, вычисленными по формулам (12). В табл. приве-

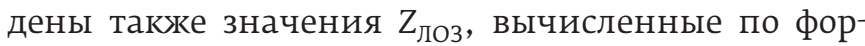
муле (11), в которой использованы значения ПДУ по (12) (для ЛИЗ марки ПАПВ использованы значения $W_{\text {пду }}^{\Sigma}$ мДж для $\lambda=532$ нм и $\lambda=1064$ нм).

В правом крайнем столбце табл. в скобках приведены значения $Z_{\text {лоз }}$, вычисленные по формуле (11), для некорректных значений Пду по СанПин.

\section{ВЫвОДЫ}

1. Применение формул (12) для вычисления значений ПДУ многоволнового лазерного излучения дает результаты меньшие и более корректные, чем полученные по формулам, регламентированным в СН 5804-91 и СанПин 2.2.4.3359-16.

2. Применение формул для вычисления ПДУ для многоволнового лазерного излучения, регламентированных в СН 5804-91 и СанПиН 2.2.4.3359-16, приводит к недопустимому занижению коэффициентов степени опасности ЛИ и занижению расстояний до границ зон безопасности, что вводит в заблуждение потребителей ЛИЗ и соответствующие контролирующие органы Роспотребнадзора и Минтруда России.

3. Необходимо внести изменения в СН 5804-91 и СанПиН 2.2.4.3359-16 в части методов вычисления значений гигиенических нормативов многоволнового лазерного излучения.

\section{ЛИТЕРАТУРА}

1. Рахманов Б. Н., Кезик В. И., Кибовский В.Т. Правила определения предельно допустимых уровней при одновременном воздействии на глаза и кожу лазерного излучения с различными длинами волн. Медицина труда и промышиенная экопогия. 2018; 12.

Rahmanov B. N., Kezik V.I., Kibovskij V.T. Pravila opredeleniya predel"no dopustimyh urovnej pri odnovremennom vozdejstvii na glaza i kozhu lazernogo izlucheniya s razlichnymi dlinami voln. Medicina truda i promyshlennaya ekologiya. 2018; 12.
2. Желтов Г. И. Нормативы по лазерной безопасности: истоки, уровень, перспективы. Фотоника. 2017; 1 ( 61): 10-35.

ZHeltov G. I. Normativy po lazernoj bezopasnosti: istoki, uroven", perspektivy. Fotonika. 2017; 1 (61): 10-35.

3. Рахманов Б. Н., Кибовский В.Т. Оценка степени опасности и ослепляющего действия лазерных изделий, работающих на открытых пространствах в видимой и ближней ИК-областях спектра. Безопасность жизнедеятепьности. Припожение. 2014; 1: 1-24. Rahmanov B. N., Kibovskij V. T. Ocenka stepeni opasnosti i osleplyayushchego dejstviya lazernyh izdelij, rabotayushchih na otkrytyh prostranstvah v vidimoj i blizhnej IKoblastyah spektra. Bezopasnost" zhiznedeyatel"nosti. Prilozhenie. 2014; 1: 1-24.

4. Будущее наступипо: эксперты рассказапи об испопьзовании пазерного оружия. URL: https://defence.ru/article/buduschee-nastupilo Budushchee nastupilo: eksperty rasskazali ob ispol"zovanii lazernogo oruzhiya. URL: https://defence.ru/article/buduschee-nastupilo

5. Истребитепь МИГ-35 оснастят пазерным оружием. URL: www. interfax.ru/russia/547292 Istrebitel' MIC-35 osnastyat lazernym oruzhiem. URL: www. interfax.ru/russia/547292

6. Сатана, кинжап и другие: какое оружие Путин показап Федерапьному собранию. URL: https://news.mail.ru/amp/politics/32719709/.

Satana, kinzhal i drugie: kakoe oruzhie Putin pokazal Federal'nomu sobraniyu. URL: https://news.mail.ru/amp/politics/32719709/.

7. Минобороны РФ: Лазерные комппексы «Пересвет» поступили на вооружение ВКС. URL: https//life.ru/новости/1136225/.

Minoborony RF: Lazernye kompleksy "Peresvet" postupili na vooruzhenie VKS. URL: https//life.ru/novosti/1136225/.

8. Многовопновый импупьсный пазер ТЕСН-263Ваsic. URL: www.laser-compact.ru/prod/263_3.html. Mnogovolnovvj impul'snyj lazer TECH-263Basic. URL: www.laser-compact.ru/prod/263_3.html

9. Зеленый пазер $200 \mathrm{~mW}$. URL: vezetmne.ru/zelenyi-laser-200mW Zelenyj lazer $200 \mathrm{~mW}$. URL: vezetmne.ru/zelenyi-laser-200mW.

10. Переносной пазерный прибор оптико-электронного противодействия ПАПB. URL: WwW. npov.ru/optico-elektronye-sredstva-upravleniya-vooruzheniem_0_39.html. Perenosnoj lazernyj pribor optiko-elektronnogo protivodejstviva PAPV. URL: www.npov.ru/optico-elektronye-sredstva-upravleniya-vooruzheniem 0 39.html.

11. Система защиты самолетов от переносных зенитных ракетных комппексов МАNTA (2012). URL: aviator.guru/blog/43730683859/sistema-zaschityi-samolyotov-otperenosnyyih-zenitnyich-kompleksov.

Sistema zashchity samoletov ot perenosnyh zenitnyh raketnyh kompleksov MANTA (2012). URL: aviator.guru/blog/43730683859/sistema-zaschityi-samolyotov-ot-perenosnyvih-zenitnyich-kompleksov.

12. На спеисамолеты КАПО установят систему пазерного противодействия переносным зенитно-ракетным комплексам. URL: https://www.business-gazeta.ru/news/62143. Na specsamolety KAPO ustanovyat sistemu lazernogo protivodejstviya perenosnym zenitnoraketnym kompleksam. URL: https://www.business-gazeta.ru/news/62143.

13. Шойгу поставип оценку "оборонке».

URL: https://utro.ru/articles/2016/10/21/1301916.shtml.

SHojgu postavil ocenku "oboronke». URL: https://utro.ru/articles/2016/10/21/1301916.shtml.

14. Лазерный проектор дпя пазерной рекламы. URL: наружная.лазерная-реклама.рф/?yelid=20917778296311777957\#4 Lazernyj proektor dlya lazernoj reklamy. URL: naruzhnaya.lazernaya-reklama. If/?yelid=20917778296311777957\#4.

15. Санитарные нормы и правипа устройства и экспиуатации пазеров № 5804-91. Утв. Зам. Гпавного государственного санитарного врача 31.07.1991.

URL: files.stroyinf.ru/Data2/1/4293847/4293847331.htm. Sanitarnye normy i pravila ustrojstva i ekspluatacii lazerov № 580491. Utv. Zam. Glavnogo gosudarstvennogo sanitarnogo vracha 31.07.1991. URL: files.stroyinf.ru/Data2/1/4293847/4293847331.htm.

16. Санитарно-эпидемиологические правипа и нормативы СанПин 2.2.4.3359-16. Санитарноэпидемиопогические требования к физическим факторам на рабочих местах. Утв. постановлением Гиавного государственного санитарного врача РФ от 21.06.2016 № 18. URL: files.stroyinf.ru/Index2/1/4293753/4293753139.htm. Sanitarno-epidemiologicheskie pravila i normativy SanPin 2.2.4.3359-16. Sanitarno-epidemiologicheskie trebovaniya $\mathrm{k}$ fizicheskim faktoram na rabochih mestah. Utv. postanovleniem Glavnogo gosudarstvennogo sanitarnogo vracha RF ot 21.06.2016 № 18. URL: files.stroyinf.ru/Index2/1/4293753/4293753139.htm.

17. Методика проведения специапьной оценки усповий труда. Утвв. приказом Минтруда Росcuu om 24.01.2014 2., № 33н.

Metodika provedeniya special"noj ocenki uslovij truda. Utv. prikazom Mintruda Rossii ot 24.01.2014 g., № 33n. 